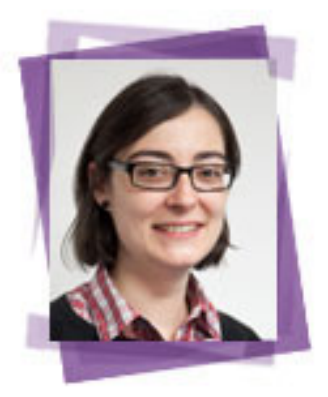

\title{
Revisión del entorno de trabajo de un traductor de contenido web
}

Anna Estellés

Grupo Tecnolettra, Universitat Jaume I, Castellón

\section{Resumen}

El objetivo de este artículo es reflexionar sobre la figura del traductor en relación con el entorno cambiante y en constante evolución en que tiene lugar la localización de contenidos web. Para ello, realizamos un repaso general sobre las novedades en la industria y a continuación, explicamos algunas de las funciones del traductor en este contexto y los tipos de herramientas que le pueden ayudar a desempeñar su trabajo.

\section{Palabras clave}

localización web, traductor de contenidos web

\section{La industria de la localización}

De acuerdo con LISA, la localización se refiere al proceso de modificar productos o servicios para adaptarlos a las diferencias de mercados distintos. Este proceso consiste, a grandes rasgos, en adaptar el producto considerando aspectos lingüísticos, físicos, técnicos, empresariales y culturales (LISA, 2007). En el caso que nos ocupa el producto viene a ser el contenido de un sitio web, por tanto debemos hablar de localización de contenido web (Mata Pastor, 2005:188-190).

Hoy en día, con la localización de contenido web como disciplina asentada, podemos analizar con más perspectiva qué futuro y presente espera al traductor profesional de proyectos web y cuáles son los retos a los que se tendrá que enfrentar.

Desde la industria de la localización, el entorno del traductor se puede contextualizar del modo descrito por Smith Jewell, CEO y fundador de Welocalize, que identifica en su blog corporativo un conjunto de aspectos en proceso de cambio que deben ayudar a mejorar el entorno de trabajo de los equipos de traducción de contenidos web (Yewell, 2010). A continuación, los vamos a ir desgranando y desarrollando con otras aportaciones.

\subsection{Estandarización}

Uno de los cambios que se observan en la industria de la traducción de páginas web es el fortalecimiento de iniciativas de estandarización que pretenden salvar las barreras impuestas por tecnologías no compatibles ni integradas. Actualmente, existen distintos formatos que permiten el intercambio de productos propios de un proceso de traducción web. XLIFF (XML Localization Interchange File Format), por ejemplo, es un formato basado en XML creado para estandarizar los ficheros de localización; TMX, es un estándar para el intercambio de memorias de traducción; SRX (Segmentation Rules Exchange) un estándar de reglas de segmentación y TBX es el estándar xml propuesto para el intercambio de bases de 
datos terminológicas. No obstante, estas iniciativas son todavía demasiado independientes y su asimilación por parte de la industria no acaba de desembocar en tecnologías integradoras que combinen un mismo formato. Esto implica que el traductor debe lidiar con distintos formatos y protocolos de intercambio de datos donde encontramos además de los ya mencionados, otros propios de herramientas TAO o TA específicas como TTX para memorias TRADOS, o TTK para proyectos en CATATYST, etc.

Otro aspecto relevante para entender el entorno del traductor es la evolución constante que existe en materia de programación web. El paso de la web 1.0 a la web 2.0 implica que el traductor se modernice y entienda las implicaciones de traducir una página web donde el contenido es creado por diferentes tipos de usuario y mediante diferentes herramientas. Se hace necesario re-adaptar el proceso de traducción a las condiciones en las que se lleva a cabo, donde la interacción con el usuario final exige celeridad y al mismo tiempo, como señala Yewell, se convierte en un agente óptimo para evaluar la calidad de la traducción.

\subsection{Cadena de producción}

La visibilidad del traductor también merece ser considerada en este contexto. El traductor de páginas web forma parte de un complejo equipo y a menudo, la toma de decisiones en las primeras fases de la localización web no conlleva su participación. Esto supone, por un lado, que el traductor deberá adaptarse a un proceso donde no siempre las cosas están pensadas para facilitar su trabajo. Por eso, resulta necesario habilitar y conocer los canales que aportan transparencia al proceso de traducción y permiten la comunicación entre cliente-equipo.

En esa línea, encontramos un 'obstáculo' cada vez más incipiente en el entorno del traductor: se trata de la constante aceleración de los tiempos de producción. La rápida evolución de la industria web conlleva que el traductor deba enfrentarse a plazos de producción muy cortos y al mismo tiempo muy exigentes. Esto promueve un trabajo colaborativo en constante reconversión. Ya no se trata únicamente de conocer las herramientas adecuadas de traducción web sino de poder hacer frente a un mercado 'hipercompetitivo' donde el trabajo en equipo es una clave esencial y donde el traductor deberá ser flexible y dinámico para encajar en el proceso de producción (Esselink, 2003:4-7) .

Esta hiper-competitividad en un mercado acelerado que cada vez exige más calidad supone, además, que el traductor aprenda a convivir con sistemas de traducción automática. Este aspecto resulta crucial para poder hacer frente a producciones con plazos muy cortos y un gran volumen de palabras (Yanishevsky, 2009:12-13).

Finalmente, hay dos aspectos vinculados directamente con la dirección de empresas de localización web que pueden considerarse contenidos transversales necesarios en la formación de traductores. Por un lado, tenemos la creciente importancia que se da a los análisis ROI en localización, es decir, cuantificar el retorno de la inversión en localización (DePalma, 2006). Esto es algo que en otras industrias es de uso generalizado y que cada vez resulta más necesario en la industria de la traducción. Nuestros traductores, por tanto, deben entender el complejo proceso empresarial en el que se mueven y conocer los mecanismos que sirven para dar cuenta del valor de su trabajo.

Por otro lado, las empresas de localización se están dando cuenta de que los clientes exigen soluciones integradas, que no pasan únicamente por la traducción sino que se completan con respuestas tecnológicas que mejoren y aceleren la localización web de sus sitios a corto y largo plazo (Esselink, 2000:13). Cada vez podemos observar más muestras de la integración de servicios web y localización en distintas empresas del sector de la traducción; algo que antes se hacía desde la industria de desarrollo web pero no a la inversa. Un ejemplo es la arquitectura multilingüe que desarrolló la empresa Linguaserve para Aena. Consiste en un sistema que combina una terminología aeronáutica muy compleja, en las áreas 
reservadas para los profesionales, con el lenguaje directo utilizado en las zonas destinadas al público en general. La tecnología desarrollada está orientada al ahorro de costes, mediante la reutilización de traducciones, así como la automatización del flujo de trabajo de mantenimiento multilingüe (Cenzano, 2005). Esta tipo de revisión del negocio implica que el traductor debe conocer cómo se pueden engranar las piezas que constituyen el desarrollo de un sitio web multilingüe y de este modo, pueda realizar mejor su trabajo.

\subsection{Formación previa}

Con este contexto como referente, podemos afirmar que el entorno de trabajo de un traductor de páginas web es un entorno cambiante y complejo que se atiene a multitud de variables (equipo del proyecto, materia prima, dificultades técnicas, dificultades lingüísticas, problemas socioculturales, tiempo, calidad, etc.). Desde las universidades y centros de enseñanza se contribuye positivamente a la formación de traductores de cara a esta realidad dinámica que rodea la traducción de páginas web. Un repaso por los distintos cursos de especialidad, máster o doctorados nacionales e internacionales nos mostrará una creciente actividad orientada a revisar y proponer nuevas metodologías de trabajo, herramientas o aplicaciones que contribuyen a la evolución de la disciplina. En estos casos, la formación viene muchas veces de la mano de profesionales reputados que en su rol como docentes nos acercan a la realidad de la industria y nos enseñan las mejores metodologías y sistemas de trabajo para afrontar la traducción web. Un ejemplo, nos conduce inequívocamente a los distintos cursos sobre localización de páginas web que imparte el profesor Manuel Mata Pastor a lo largo del territorio nacional. En este tipo de cursos, presentes en másters como el de Tradumática (Universitat Autónoma de Barcelona) o Tecnoloc (Universitat Jaume I), se profundiza en las variadas facetas del traductor de páginas web, las distintas fases del proceso de traducción así como otros aspectos más técnicos o lingüísticos relevantes para el perfil.

Además, otra forma de mantenerse al día es consultar en Internet distintas páginas especializadas que analizan la evolución de la industria (como esta revista o blogs y boletines de empresas de localización) o recogen las últimas novedades acerca de las herramientas de localización. Un ejemplo sobre herramientas de licencia libre es la página que dirige Silvia Flórez: 'Traducción y Mundo Libre' (Flórez Giraldo, 2009) que recoge algunos de los resultados de su trabajo de investigación (Flórez Giraldo, 2008) y donde el traductor profesional puede informarse sobre las últimas novedades en tecnologías de la traducción.

A continuación, sin perder de vista el macro-contexto de la industria de la localización, vamos a examinar las distintas funciones del traductor, sus roles y las herramientas que tiene al alcance para desarrollar las múltiples tareas en las que se ve involucrado durante el proceso de traducción de contenidos basados en la web 2.0.

\section{Identificando la materia prima}

El proceso de la localización de contenido web forma parte de un proceso más amplio que ha venido a llamarse globalización. Tal y como lo define la asociación LISA (LISA, 2007:11), la globalización de productos engloba el proceso de internacionalización y el de localización. La internacionalización de un producto consiste en prepararlo/desarrollarlo de modo que pueda ser transportado a otros contextos lingüístico-culturales. Por eso, si el proceso de internacionalización es positivo, durante la localización resultará más fácil extraer los fragmentos traducibles de la amalgama de código de estructura, applets, etc. Por ejemplo, el contenido web de un sitio que ha sido desarrollado en php y MySQL, tendrá la materia prima almacenada, en su mayoría, en la base de datos aunque también habrá que traducir fragmentos de texto de las plantillas en php u otros archivos de tipo media o de soporte a la web. Una buena internacionalización previa, supone, entre otros aspectos, que la información contenida en la base de 
datos se haya etiquetado para distinguir el idioma o que hayamos diferenciado entre ficheros php que forman parte de la cara visible del sitio (lo que ve el usuario) y ficheros que forman parte del sistema de gestión interno del contenido y no es necesario traducir. Se trata de haber establecido en el proceso de internacionalización, qué fragmentos son candidatos a ser traducidos y cuáles no. Además, resultará de gran utilidad haber preparado los ficheros gráficos, animaciones, etc. de cara a una posible traducción. Un buen proceso de internacionalización es fundamental y existen numerosas herramientas para verificar lo 'internacionalizado' que está un sitio web, un ejemplo gratuito es la aplicación que se ha desarrollado recientemente dentro de la W3C (W3C, 2010).

En definitiva, si la internacionalización ha dado los frutos esperados y el análisis del contenido web está claro, la materia prima del traductor serán principalmente ficheros .html o .xhtml, o el formato PO (ficheros .po) en caso de plataformas que hacen uso de gettext (FSF, 2010), sistema utilizado en plataformas y aplicaciones basadas en software libre (FSF, 2007).

Un fichero html contiene código html y texto traducible. Un fichero .xhtml es muy similar a un html sólo que más complejo. En ambos casos se trata de lenguajes en evolución, con lo que podemos encontrar distintas versiones dentro del mismo lenguaje, por eso resulta interesante consultar las especificaciones de cada versión en la página de la W3C (www.w3.org), que es la organización que se encarga de su estandarización. En lo relativo al tipo de código que contienen, ambos suelen contener etiquetas para indicar la estructura y no encontraremos demasiados aspectos de formato, ya que éstos suelen estar declarados en un fichero diferente cuya extensión suele ser .css. Un fichero .css no suele traducirse pero debemos conocerlo porque puede ser útil si tenemos problemas de formato. En el caso de plataformas cuya traducción se basa en gettext, el fichero .po es el resultado de un proceso de extracción de texto traducible (Fernández García, 2006, 79-86).

Además de estos ficheros, podemos encontrarnos con ficheros gráficos, animaciones, videos o pequeños trozos de código que hacen referencia a mini-aplicaciones dentro de la Web (javascript, ajax...) o a la plataforma web (php, asp...). La forma de organizar todos estos ficheros supone uno de los principales retos del proceso de localización (Mata Pastor, 2005: 201). En los casos en los que el contenido de la web se descarga para ser localizado, el traductor deberá acostumbrarse a trabajar con sistemas de carpetas jerarquizados que contienen la materia prima a traducir organizada por capas. No obstante, hoy en día, puede darse la situación de que la localización se haga directamente al servidor, de modo que no vamos a disponer físicamente de todos estos ficheros para manipularlos. Este enfoque está presente en sitios webs desarrollados a partir de un sistema de gestión de contenido multilingüe o global, donde el grueso de la traducción se realiza a distancia mediante plataformas virtuales on-line que graban los datos directamente en el servidor, aplicaciones como ShadoCMS y su Translation API (Straker, 2009) son un ejemplo de ello aunque existen alternativas de licencia libre como Drupal y su cliente de localización (Buytaert, 2007).

No debemos olvidar la tendencia a utilizar el formato estándar de localización XLIFF que sirve para aglutinar cadenas de texto a traducir que originalmente estarían en distintos formatos (Savourel, 2001: 377-395). XLIFF tiene como propósito almacenar el contenido traducible de un sitio y facilitar su intercambio (Savourel, Reid y otros, 2008).

Finalmente, es necesario recordar que el entorno de un traductor de contenido web puede incluir situaciones comunicativas muy diversas. Con esto, queremos decir que el lenguaje utilizado en los contenidos puede de diversas especialidades (lenguaje general, médico, jurídico...); el uso de recursos terminológicos y lingüísticos estará a la orden del día en este contexto. 


\section{El rol del traductor con respecto a su entorno: recursos y herramientas}

La localización de contenido web es un proceso complejo en el que tienen lugar distintos subprocesos cuya naturaleza difiere según el contexto, el propósito, etc. El tamaño del proyecto o el equipo humano/tecnológico que la empresa de localización destine para realizarlo son aspectos que influirán directamente en los roles que tendrá que desempeñar el traductor. Por ejemplo, si hablamos de un sitio pequeño no demasiado complicado es posible que el traductor se encargue de todo el proceso (Mata, 2005: 237) pero si éste no es el caso, el traductor será un agente más que participará en el proceso. Díaz Fouces (Díaz Fouces, 2004:29) describe el ciclo operativo del proceso de localización basándose en los siguientes puntos:

1. Recepción y gestión del pedido

2. Organización de recursos humanos

3. Organización de recursos materiales

4. Traducción/Localización

5. Control de calidad

6. Integración de contenidos

7. Evaluación

8. Entrega

Si nos atenemos al plano meramente lingüístico y comunicativo, el traductor tiene un papel principal en la fase de control terminológico, traducción de texto y control de la calidad. Sin embargo, el papel del traductor en otras fases del proceso supone ventajas sustanciales que repercuten directamente en el proceso, por ejemplo, mejorando los tiempos de producción o aumentando la calidad del proyecto.

Por otra parte, un elemento crucial del entorno del traductor es el uso de herramientas informáticas que le ayuden a desarrollar las distintas tareas. Las herramientas o tecnologías que puede utilizar un traductor son muy variadas. Basándonos en la tipología que recoge Alcina (Alcina, 2008:96-99) se distinguen los siguientes tipos:

- tecnología del equipo informático, como los componentes físicos, el sistema operativo, antivirus, etc.

- herramientas de comunicación y documentación, como clientes de correo, video chat, base de datos bibliográficas, enciclopedias online, etc.

- herramientas de edición textual y maquetación para editar texto, imágenes, vídeo etc.

- herramientas lingüísticas y recursos, como glosarios, memorias de traducción, etc.

- herramientas de traducción entre las que se distinguen las herramientas de traducción asistida, herramientas de traducción automática, híbridos, etc.

El entorno del traductor de contenidos web se completaría con herramientas de gestión de proyectos que ayudan a sistematizar, administrar y controlar los procesos de localización (Esselink, 2000: 427).

A continuación, vamos a analizar el rol del traductor atendiendo a sus distintas funciones en el proceso pero además, iremos añadiendo información sobre las tecnologías de las que puede hacer uso.

\subsection{Recepción y gestión del encargo}

Es la fase que sigue a la pre-venta. En esta fase se lleva a cabo el análisis del contenido del encargo, se determinan los detalles a tener en cuenta de cara al presupuesto, el método de obtención de los contenidos a traducir y la clasificación del material de acuerdo con su formato, especialidad, etc.

Durante esta fase el traductor trabajará muy estrechamente con el personal informático y el gestor del proyecto. El objetivo es identificar el texto traducible y calcular las posibilidades de extracción y localización. Se analizará todo el contenido, para identificar problemas de traducción de tipo lingüístico, comunicativo o técnico con el fin de asegurar que el presupuesto se adecue al esfuerzo que se deberá 
realizar después. Se realizarán recuentos de palabras, clasificación por tipos de lenguaje (niveles de especialidad que contiene el sitio a traducir), por tipos de formato origen (imágenes traducibles, texto en código, etc.) y cualquier otro aspecto que pueda suponer un esfuerzo extra durante el proceso de localización. Todo esto resulta imprescindible para poder distribuir adecuadamente la carga de trabajo en los tiempos exigidos.

Las herramientas a utilizar serán aquellas que nos ayuden a preparar la gestión del proyecto. Es común que nos encontremos con contadores de palabras integrados en paquetes de software como SDL TRADOS STUDIO (SDL, 2009), independientes como CatCount (AIT, 2009) o TKount (Prior, 2009) o software específico de cada empresa.

Para obtener los contenidos a traducir existen distintos mecanismos que dependerán de las posibilidades del cliente y del servicio utilizado. A veces, basta con un sistema de transferencia de archivos por Ftp, otras el gestor de contenidos integrado con el sistema de gestión de la traducción nos permite obtener paquetes con las cadenas traducibles. Un ejemplo es la plataforma FreeWay de LionBridge que se integra con distintos gestores de contenido para permitir el flujo de traducción. Otros gestores permiten hacer esto de una manera más directa, por ejemplo, SDL Tridion funciona como un gestor de contenido multilingüe que integra herramientas para la traducción de contenido web. Normalmente, el traductor no es el encargado de realizar esta tarea pero sí que le puede resultar útil conocer estas herramientas para extraer contenido con fines documentales, por ejemplo. La gestión de imágenes y contenido media traducido se puede realizar mediante sistemas de licencia libre como Image Localization Manager (Muñoz Sánchez, 2008) o privativos como WebBudget XT (Aquino, 2009).

Además, es posible que el traductor deba determinar el grado de especialización del contenido web. En este aspecto, puede ser de ayuda utilizar herramientas de tipo lingüístico como analizadores de corpus, por ejemplo, WordSmith (Scott, 2005) que permiten extraer las palabras clave de un corpus.

\subsection{Organización de los recursos humanos}

Durante esta fase el traductor se organiza dentro del equipo de trabajo. Se establecerán los tiempos del proyecto y se planificarán las tareas a realizar, asignándolas a los distintos miembros del equipo. Para ello, se establecerá el número de agentes implicados en el proceso atendiendo a la información recopilada en la fase anterior y las observaciones técnicas o lingüísticas específicas del encargo.

Las herramientas que predominan en esta fase son de tipo gestión de proyectos, como por ejemplo Project Professional (Microsoft, 2010) o MindManager (MindJet, 2008); también es frecuente que el flujo de trabajo se controle mediante documentos más rudimentarios compartidos en el sistema de carpetas del proyecto o mediante plataformas web que sirven para gestionar el flujo de trabajo entre traductores que no están en plantilla, proveedor y equipo en plantilla. Este tipo de herramientas suelen ser ad hoc con el fin de adaptarse al sistema de producción de cada empresa, aunque existen ejemplos genéricos y con licencia GNU/GPL como PHProjekt (MayFlower, 2010) o Project-Open (ProjectOpen, 2010).

Finalmente, cabe señalar la repercusión de esta fase en las siguientes, ya que la elección o viabilidad de los recursos humanos disponibles para un encargo influirá en aspectos posteriores como, por ejemplo, si se va a hacer uso de herramientas de traducción automática.

\subsection{Organización de los recursos materiales}

El traductor cotejará sus recursos documentales con el contenido de la web. Dentro de esta fase, el traductor debe empezar la tarea de documentación. Las herramientas más comunes durante esta fase son aquellas aplicaciones que permiten la comunicación entre el traductor y otros miembros del equipo 
(clientes de correo electrónico, etc.) y también herramientas de documentación que nos permiten localizar documentos y recursos documentales relativos al contenido web, por ejemplo, documentos del campo de especialidad, textos paralelos susceptibles de formar parte de una memoria de traducción de apoyo o recursos reutilizables de otros proyectos.

Entre las herramientas de documentación, encontramos las bases de datos terminológicas, como Multiterm (SDL, 2009), que nos permiten recuperar terminología de otros proyectos. Además, utilizaremos herramientas de gestión de memorias de traducción para verificar si tenemos cadenas traducidas y reutilizarlas. Es útil que el traductor conozca iniciativas en localización y traducción general que permiten el intercambio de memorias de traducción para su explotación, como por ejemplo, la iniciativa TAUS (TAUS, 2004) está orientada a la explotación de recursos para la traducción automática y permite a grandes y medianas empresas el acceso compartido a memorias de traducción (formato TMX).

Para organizar y controlar los recursos podemos hacer uso de herramientas de gestión documental, como DocuGes (Pérez Vidal, 2010), para controlar tanto los recursos terminológicos existentes (diccionarios, bases de datos...) como memorias de traducción, hojas de estilo, etc. También podemos utilizar un gestor de recursos como Zotero (Stillman, Kornblith y otros, 2010) que permite organizar recursos online y físicamente desde el ordenador.

Toda esta información se recogerá en el sistema de gestión del proyecto de modo que todos los miembros del equipo puedan beneficiarse de ella. Además, estos recursos reutilizables se utilizan también en fase de pre-localización, cuando hacemos uso de herramientas de traducción automática.

\subsection{Traducción/localización}

La fase de traducción es la parte del proceso donde el traductor es el actor principal. Durante esta fase, llevará a cabo la traducción de todo el texto traducible, incluyendo aquel que habrá sido extraído de imágenes, animaciones o aplicaciones web.

Existen numerosas herramientas que nos ayudan a llevar a cabo la labor, entre ellas, distinguimos dos grandes grupos: las herramientas de traducción automática que suelen utilizarse al inicio del proceso y las herramientas de traducción asistida que completan el proceso de traducción.

En el primer grupo encontramos herramientas como Systran (Systran, 2007) que combinan el uso de memorias de traducción con motores de traducción automática. Además esta herramienta permite extraer glosarios terminológicos que se podrán reutilizar más tarde en el proceso de traducción asistida.

En el segundo grupo, encontramos herramientas como GlobalSight (WeLocalize, 2009), que permite no sólo la traducción asistida del contenido sino la gestión del flujo de trabajo y los contenidos del proyecto. Otras herramientas como CatsCradle (Spencer, 2009) o Webbudget (Aquino, 2009) suponen alternativas interesantes si hablamos de sitios web de tamaño reducido. En la línea de aplicaciones integradas como GlobalSight encontramos también SDL Tridion (SDL, 2009), una herramienta que permite desde la gestión de contenido web a la interacción con otras aplicaciones TRADOS.

En lo que refiere a la traducción de archivos .po podemos utilizar distintos sistemas, si la traducción se realiza directamente al servidor, podemos hacer uso de plataformas como Launchpad (Canonical, 2010) o CrowdSight (WeLocalize, 2009) que funcionan como plataforma de trabajo colaborativo, donde se incluye el proceso de localización, y permiten gestionar el flujo de traducción de un sitio web. Asimismo, a nivel escritorio encontramos herramientas como PoEdit (Slavik, 2010) que permite editar ficheros .po de un modo limpio y funcional, de forma que el traductor no tenga que trabajar directamente sobre el código. 


\subsection{Control de calidad}

La fase de control de calidad constituye el momento en el que se revisan aspectos lingüísticos, comunicativos, técnicos y ergonómicos. Las herramientas de revisión de aspectos lingüísticos y comunicativos se incluyen a menudo dentro de las mismas aplicaciones de traducción en forma de utilidades de edición textual y permiten revisar errores de traducción, orto-tipografía o gramática y también asegurar la consistencia terminológica. CrowdSight (WeLocalize, 2009) por ejemplo, permite aplicar controles de calidad en distintas partes del flujo de producción cuando trabajamos con el sistema de traducción de GlobalSight (WeLocalize, 2009).

En lo que refiere a los aspectos técnicos y ergonómicos, esta tarea suele correr a cargo del personal informático, que verificará el funcionamiento del sitio web. Una herramienta de licencia abierta que permite verificar que los enlaces de nuestro sitio funcionan es LinkChecker (Kleneidam, 2010).

\subsection{Integración de contenidos}

En la fase de integración, el traductor tiene funciones de apoyo, por ejemplo, en caso de que el personal técnico detecte alguna inconsistencia o tenga problemas asociados a la traducción que impidan la integración de los contenidos.

\subsection{Evaluación}

En la fase de evaluación el traductor verificará que el contenido está bien traducido y que se puede visualizar todo correctamente. Es frecuente que las pruebas se realicen desde distintos navegadores (MS Explorer, Mozilla Firefox...) y a distintas resoluciones para asegurar que el producto generado cumple con las mismas expectativas que el original.

\subsection{Entrega}

La fase de entrega incluye la entrega del encargo, su facturación y la recogida de contenidos reutilizables. El traductor participa activamente de esta última parte. Deberá recoger y almacenar los recursos generados (glosarios, memorias de traducción, protocolos para solventar problemas surgidos, hojas de estilo revisadas, etc.) de forma que se puedan localizar en el sistema de gestión documental de la empresa en el futuro. Nuevamente, haremos uso de herramientas documentales que nos permiten organizar los recursos generados. Asimismo es posible que utilicemos algún gestor de memorias de traducción para agregar los nuevos segmentos a memorias ya existentes o un gestor de bases de datos terminológicas para reorganizar nuestra terminología.

En cuanto a la facturación, si el proyecto es pequeño podemos utilizar herramientas de gestión como Webbudget (Aquino, 2009), aunque normalmente, en empresas con gran facturación, nos encontraremos con módulos específicos ad hoc integrados o no el sistema de traducción.

Una valor añadido al proceso de localización puede ser la realización de un análisis ROI (retorno en inversión) para valorar las ventajas económicas y de marketing que tiene el cliente al haber localizado el producto (DePalma, 2006). El traductor no es un agente que participe activamente de este proceso, pero le será de utilidad saber que existen sistemas de medida y mecanismos disponibles para poder evaluar las ventajas de un sitio web localizado con respecto a uno no localizado. Herramientas genéricas como contadores o rastreadores de visitas o formularios de satisfacción son ejemplos humildes al alcance de todos. AWstats (Destailleur, 2009), por ejemplo, permite obtener estadísticas sobre la localización geográfica del usuario, entre otros aspectos. 


\section{Conclusiones}

Con este artículo, pretendíamos dar una visión general de lo complejo y variable que es el entorno del traductor de contenido web. Como conclusión, nos gustaría hacer hincapié en el reciclaje constante que exige formar parte de esta industria en continuo crecimiento y evolución. La formación a través de másters, como los que imparte la Universidad Autónoma de Barcelona (CITA) o la Universidad Jaume I (CITA) o cursos de especialización (como los que ofrecen las propias empresas de desarrollo de software específico, por ejemplo, TRADOS) resultarán de gran utilidad al traductor.

\section{Referencias}

\section{Enlaces a herramientas citadas}

AIT (2009). CatCount.

http://www.catcount.com/. Fecha de consulta: 27-06-2010.

Aquino. (2009). Webbudget XT.

http://www.webbudget.com/. Fecha de consulta: 27-06-2010.

Buytaert, D. (2007). Drupal Localization Client.

http://drupal.org/project/l10n client. Fecha de consulta: 27-06-2010.

Canonical (2010). LaunchPad.

https://launchpad.net/. Fecha de consulta: 27-06-2010.

Destailleur, L. (2009). AWStats.

http://awstats.sourceforge.net/. Fecha de consulta: 27-06-2010.

Kleneidam,B. (2010). LinkChecker.

http://linkchecker.sourceforge.net/ . Fecha de consulta: 27-06-2010.

MayFlower (2010). PhpProjekt, MayFlower GmhbH.

http://www.phprojekt.com/. Fecha de consulta: 27-06-2010.

Microsoft (2010). Project Professional, Microsoft.

http://www.microsoft.com/project/. Fecha de consulta: 27-06-2010.

MindJet (2008). MindManager.

http://www.mindjet.com/products/mindmanager-8-win/overview. Fecha de consulta: 27-06-2010.

Muñoz Sánchez, P. (2008). Image Localization Manager.

http://sourceforge.net/projects/ilmanager/. Fecha de consulta: 27-06-2010.

ProjectOpen (2010). ProjectOpen, Barcelona.

http://www.project-open.com/. Fecha de consulta: 27-06-2010.

Pérez Vidal, F. J. (2010). DocuGes. 
http://docuges.sourceforge.net/. Fecha de consulta: 27-06-2010.

Prior, M. (2009). TKount.

http://www.omegat.org/es/resources.html. Fecha de consulta: 27-06-2010.

Scott, M. (2005). WordSmith 5.

http://www.lexically.net/wordsmith/. Fecha de consulta: 27-06-2010.

SDL (2009). SDL Multiterm 2009

http://www.sdl.com/en/products/terminology-management/multiterm.asp. Fecha de consulta: 27-06-2010.

SDL (2009). SDL TRIDION 2009.

http://www.sdltridion.com/products/sdltridion2009/. Fecha de consulta: 27-06-2010.

SDL (2009). SDL TRADOS STUDIO 2009

http://www.sdl.com/en/sites/sdl-trados-solutions/default.asp. Fecha de consulta: 27-06-2010.

Slavik, V. (2010). PoEdit.

http://www.poedit.net. Fecha de consulta: 27-06-2010.

Spencer, J. (2009). Catscradle.

http://www.stormdance.net/software/catscradle/overview.htm. Fecha de consulta: 27-06-2010.

Stillman, D., S. Kornblith, y otros (2010). Zotero.

http://www.zotero.org/. Fecha de consulta: 27-06-2010.

Straker (2009). ShadoCMS.

http://www.shadocms.com/. Fecha de consulta: 27-06-2010.

Systran (2007). Systran Premium Translator.

http://www.systransoft.com/. Fecha de consulta: 27-06-2010.

W3C. (2010). Prototype Internationalization Checker

http://www.w3.org/blog/International/2010/06/23/prototype internationalization checker a . Fecha de consulta: 27-06-2010.

WeLocalize (2009). CrowdSight.

http://www.welocalize.com/english/technology/crowdSight.aspx. Fecha de consulta: 27-06-2010.

WeLocalize(2009). GlobalSight, Sourceforge.

https://sourceforge.net/projects/globalsight/. Fecha de consulta: 27-06-2010.

\section{Bibliografía}

Alcina, A. (2008). "Translation technologies: Scope, tools and resources". TARGET, John Benjamins. 1: 79-102.

Cenzano, A. (2005). Linguaserve diseña un portal para mejorar la oferta internacional de AENA.

Cincodías. Madrid. 
DePalma, D. A. (2006). "Quantifying the return on localization investment". Perspectives on Localization. K. J. Dunne. Ámsterdam/Filadelfia, John Benjamins.

Díaz Fouces, Ó. (2004) A Localização de Páginas da Internet na Formação de Tradutores. Confluencias: http://confluencias.net/n1/fouces.pdf.

Esselink, B. (2000). A Practical Guide to Localization. Ámsterdam/Filadelfia, John Benjamins.

Esselink, B. (2003) The Evolution of Localization. Guide to Localization: 4-7.

Fernández García, J. R. (2006). "La traducción de software: trabajo en equi/po". Linux Magazine (20). http://www.linux-magazine.es/issue/20/Educacion.pdf. Fecha de consulta: 27-06-2010

Flórez Giraldo, S. (2008). Software libre y freeware para el traductor: catálogo de herramientas y adaptación de una metodología para su evaluación con miras a un uso pedagógico. Máster oficial interuniversitario en Tecnologías de la traducción y Localización. Castellón, Universitat Jaume I, Universitat de València y Universitat d'Alacant.

Flórez Giraldo, S. (2009). Traducción y mundo libre.

http://traduccionymundolibre.com/. Fecha de consulta: 27-06-2010.

FSF (2007). GNU General Public Licence. F. S. Foundation. Boston. http://www.gnu.org/licenses/gpl.html. Fecha de consulta: 27-06-2010.

FSF. (2010). GNU Gettext. http://www.gnu.org/software/gettext/. Fecha de consulta: 27-06-2010.

LISA (2007). The Globalization Industry Primer, The Localization Industry Standards Association.

Mata Pastor, M. (2005). "Localización y traducción de contenido web". Traducción y localización: mercado, gestión y tecnologías. D. Reinecke. Las Palmas de Gran Canaria, Anroart Ediciones.

Savourel, Y. (2001). XML Internationalization and Localization. Indianápolis, Sams Publishing.

Savourel, Y., J. Reid, y otros (2008). OASIS Standard: XLIFF Versión 1.2, OASIS.

http://docs.oasis-open.org/xliff/xliff-core/xliff-core.html. Fecha de consulta: 27-06-2010.

TAUS. (2004). <www.translationautomation.com . Fecha de consulta: 27-06-2010.UAB (2010). Máster de Tradumática: Traducción y localización (IX edición) Barcelona, Universitat Autónoma de Barcelona. http://www.fti.uab.es/pg.tradumatica/Tradumatica ES/index.html. Fecha de consulta: 27-06-2010

UJI (2010). Master Tecnoloc: Tecnologías de la Traducción y Localización, Castellón de la Plana, Grupo Tecnolettra, Universitat Jaume I.

http://tecnolettra.uji.es/es/?page id=39. Fecha de consulta: 27-06-2010

W3C: Consorcio World Wide Web. http://w3.org. Fecha de consulta: 27-06-2010.

Yanishevsky, A. (2009) The Emerging Role of Machine Translation. Guide to Localization, 12-13. http://www.multilingual.com/downloads/screenSupp103.pdf. Fecha de consulta: 27-06-2010. 
$\gg>$ revista tradumàtica

Traducció i Tecnologies de la Informació i la Comunicació

Número 08: Localització i web : desembre 2010 :

http://wrww.fti.uab.cat/tradumatica/revista : ISSN: 1578-7559

Yewell, S. (2010) Our Rapidly Evolving Industry. http://welocalize.blogspot.com/2010/05/our-rapidlyevolving-industry.html. Fecha de consulta: 27-06-2010. 